\title{
Carboplatin-Pembrolizumab-Pemetrexed Regimen
}

National Cancer Institute

\section{Source}

National Cancer Institute. Carboplatin-Pembrolizumab-Pemetrexed Regimen. NCI

Thesaurus. Code C140674.

A regimen consisting of carboplatin, pembrolizumab and pemetrexed that can be used in the treatment of non-small cell lung cancer (NSCLC). 